\title{
Statistical method for thickness measurement of amorphous objects
}

\author{
D. Van Dyck and M. D. Croitoru ${ }^{a)}$ \\ EMAT, University of Antwerp, Groenenborgerlaan 171, B-2020 Antwerp, Belgium
}

(Received 19 March 2007; accepted 22 May 2007)

\begin{abstract}
The authors propose a nondestructive method for the determination of the thickness of an amorphous sample. This method is based on the statistics of the phase of the electron exit wave function, which depend on the number of atoms traversed by the incident electron which itself is a function of the thickness of the object. The accuracy of this method has been checked numerically by the multislice method and compared with that based on the mean inner potential. (C) 2007 American Institute of Physics. [DOI: 10.1063/1.2749184]
\end{abstract}

In many applications of amorphous thin objects an accurate knowledge of the thicknesses is needed because a number of physical and technological properties of thin objects strongly depend on the confinement governed by the object thickness. The control of amorphous film thickness is a necessary condition for continued device miniaturization and is indispensable for application of physical phenomena emerging in thin films. For instance, in nanoelectronics the present limit of the reduction in the size of components is the thickness of the amorphous dielectric layers, so the determination of this thickness is an important metrological challenge. In materials science in situ object thickness monitoring is often essential for characterizing the experiments. In the bright field imaging mode of the transmission electron microscope the images sensitively depend, besides other factors, on specimen thickness, which makes the accurate object thickness determination essential for the correct interpretation of the experimental results.

Thickness measuring techniques can be classified as destructive and nondestructive. The most used destructive methods employ cross section in a scanning electron microscope, cross section in a transmission electron microscope, ${ }^{1}$ electron impact emission spectroscopy, ${ }^{2}$ reflection highenergy electron diffraction, ${ }^{3}$ sputter depth profiling, and $\mathrm{x}$-ray photoelectron spectroscopy, ${ }^{4}$ while among nondestructive techniques we mention $\mathrm{x}$-ray diffraction, ${ }^{5} \mathrm{x}$-ray fluorescence spectroscopy, ${ }^{5,6}$ spectroscopic ellipsometry, ${ }^{7}$ Rutherford backscattering, nuclear reaction analysis, and energy dispersive spectroscopy. 8,9 A simple method of mass thickness determination for amorphous and polycrystalline films based on the Rutherford approximation is proposed in Ref. 10. However, there is a strong necessity to develop a thickness measurement technique, which will be fast, cheap, and nondestructive, and can be applied to amorphous films with of light elements.

The basic idea behind our approach is as follows. Since the phase shift through an individual atom can be calculated, the distribution of the phase shifts at the exit face of the amorphous object can be expressed in statistical terms as a function of the number of atoms that are crossed, which itself is a function of the object thickness. If we assume that the object consists of atoms of the same type, with empty space in between, we can calculate the statistics of the phase of the exit wave from which we can determine the average number

\footnotetext{
${ }^{a)}$ Electronic mail: mihail.croitoru@ua.ac.be
}

of atoms crossed by the electron beam and hence also the specimen thickness in the direction of the electron propagation. The phase of the exit wave function can be determined accurately using off axis holography with a $C_{s}$ corrected microscope. ${ }^{11}$ We assume that (i) the ray paths are straight and perpendicular to the foil, (ii) inside the atom, the phase is determined by the electrostatic potential projected along the beam, (iii) in between the atoms, the potential is constant $V_{0}$ (which might be zero), and (iv) the thickness is constant over the area of measurement.

The phase shift through the atom depends on the position of the beam $\mathbf{R}$ and is given by

$$
\varphi(\mathbf{R})=\beta \int V(\mathbf{R}, z) d z,
$$

where $\beta=\beta\left(\lambda, E_{0}\right)$ is the interaction parameter. ${ }^{12}$ Here $\lambda$ is the electron wavelength in vacuum and $E_{0}$ is the kinetic energy of the electron in vacuum. We limit the size of the atom to $R \leqslant R_{0}$ (to avoid divergences later).

Let us consider a section of the object with area $S$ and thickness $t$. If we introduce $\rho$ the mass density of material, $m$ the mass of an individual atom, and $n$ the number of atoms in the section, then the number of atoms per unit area as seen by the incident electron is

$$
D=\frac{n}{S}=\frac{\rho}{m} t
$$

Let us now consider one particular ray path $i$, as seen along the beam direction. On propagating through the object, the path crosses different atoms. The number $N$ of atoms that are crossed can be estimated as follows. If we call $R_{0}$ the radius of each atom, then the ray $i$ crosses all atoms whose origins are within a circle with radius $R_{0}$ around $i$. Thus $N=D \pi R_{0}^{2}$. Every atom crossed by $i$ adds a contribution to the phase, given by Eq. (1).

We can now reconsider the situation from another perspective. Since all atoms are identical (can be generalized), the phase of the ray path can be considered as the sum of phase contributions sampled at $N$ values $\varphi\left(R_{j}\right)$ at $N$ different positions $j=1, \ldots, N$ of the same atom. According to the central limit theorem the total phase at the exit face of the sample is then 


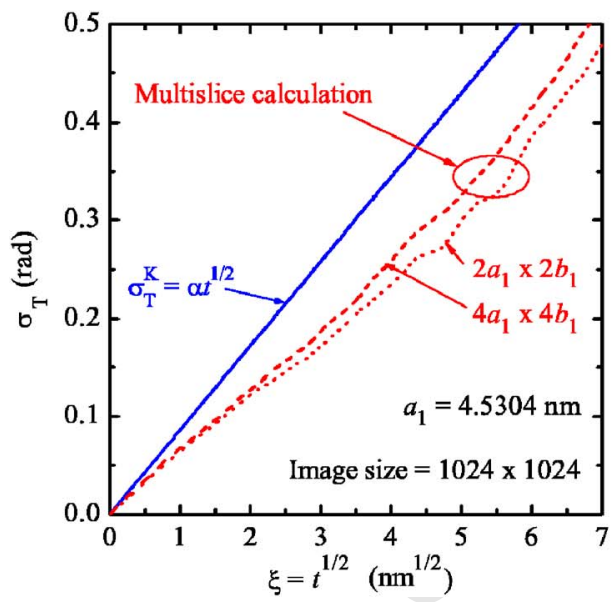

$$
\varphi_{T}=\sum_{j=1}^{N} \varphi\left(R_{j}\right)=N\langle\varphi\rangle=\frac{\rho}{m} t \pi R_{0}^{2}\langle\varphi\rangle,
$$

with $\langle\varphi\rangle=\sum_{j=1}^{N} \varphi\left(R_{j}\right) / N$.

Let us now calculate the standard deviation in $\varphi_{T}$. If we assume the samples $R_{j}$ to be uncorrelated, we have, according to the central limit theorem, $\sigma_{T}^{2}=N \sigma^{2}$, where $\sigma$ is the standard deviation of each sample $\varphi\left(R_{j}\right)$. Therefore,

$$
\sigma_{T}=\sqrt{\frac{\rho}{m} t \pi R_{0}^{2}} \sigma .
$$

Since $\varphi(R)$ only depends on the distance $R$ from the origin of the atom we can also write $\langle\varphi\rangle$ as

$$
\langle\varphi\rangle=\int_{0}^{R_{0}} \varphi(R) p(R) d R,
$$

with $p(R)$ the probability to find a distance between $R$ and $R+d R$, which is proportional to the area $p(R) d R$ $=2 \pi R d R / \pi R_{0}^{2}$. Taking into account the finite radial size of an atomic potential we get

$$
\begin{aligned}
& \lim _{R_{0} \rightarrow \infty}\langle\varphi\rangle R_{0}^{2}=2 \int_{0}^{\infty} \varphi(R) R d R=C_{1}, \\
& \lim _{R_{0} \rightarrow \infty} \sigma^{2} R_{0}^{2}=2 \int_{0}^{\infty} \varphi^{2}(R) R d R=C_{2} .
\end{aligned}
$$

Using Eqs. (3) and (4) we obtain analytical expression for the mean phase of the exit wave function in the form

$$
\varphi_{T}=\frac{\pi \rho C_{1}}{m} t
$$

and for the standard deviation of the phase of the exit wave function in the form

$$
\sigma_{T}=\sqrt{\frac{\pi \rho C_{2}}{m}} t^{1 / 2} .
$$

The parameters $C_{1}$ and $C_{2}$ can be easily calculated from the tabulated values of the scattering amplitudes. In this work we have chosen Kirkland's fitting form for the scattering amplitudes $^{12}$ that has the appropriate form at large and small scattering angles
FIG. 1. (Color online) Standard deviation and mean exit wave phase as a function of square root of the amorphous Si-sample thickness and amorphous Si-sample thickness, respectively, due to Wooten et al. with 4096 atoms in the unit cell. In both panels the solid line demonstrates the results of our theoretical model. In the right panel dashed-dotted lines exhibit results obtained from the experiment and the Hartree-Fock calculations of MIP.

where $N_{L}=3$ is the number of Lorenzians and $N_{G}=3$ is the number of Gaussians. Since the atomic potential $V(\mathbf{R}, z)$ is a three-dimensional Fourier transform of the scattering amplitudes in Born approximation, together with Eq. (1) this leads to

$$
\varphi_{T}=\gamma \beta \frac{\pi \rho}{m}\left(\sum_{i=1}^{N_{L}} \frac{a_{i}}{b_{i}}+\sum_{i=1}^{N_{G}} c_{i}\right) t,
$$

where $\gamma=2 a_{0} e\left(a_{0}=\hbar^{2} / m_{e} e^{2}\right.$ is the Bohr radius, $m_{e}$ is the rest mass of the electron, and $e$ is the charge of the electron). The standard deviation of the phase of the exit wave function is given by

$$
\begin{aligned}
\sigma_{T}= & \beta \gamma \pi\left(\frac{\pi \rho}{m}\right)^{1 / 2}\left[\sum_{i, j=1}^{N_{L}} \frac{a_{i} a_{j}}{b_{i}-b_{j}} \ln \left(\frac{b_{i}}{b_{j}}\right)\left(1-\delta_{i, j}\right)+\frac{a_{i}^{2}}{b_{i}} \delta_{i, j}\right. \\
& +\sum_{i, j=1}^{N_{G}} \frac{c_{i} c_{j}}{d_{i}+d_{j}} \\
& \left.+2 \sum_{i=1}^{N_{L}} \sum_{j=1}^{N_{G}} a_{i} c_{j} \exp \left(b_{i} d_{j}\right) \Gamma_{0}\left(b_{i} d_{j}\right)\right]^{1 / 2} t^{1 / 2}
\end{aligned}
$$

where $\Gamma_{a}(x)$ is the incomplete gamma function. ${ }^{13}$ Our calculations give for the coefficients $C_{1}$ and $C_{2}$ from Eqs. (6) and (7) the following result $\left(\underline{E_{0} / e}=300 \mathrm{kV}\right): C_{1, \mathrm{Si}}=0.05808 \AA^{2}$, $C_{1, \mathrm{C}}=0.02499 \AA^{2}, \quad \sqrt{C_{2, \mathrm{Si}}}=0.06890 \AA$, and $\sqrt{C_{2, \mathrm{C}}}$ $=0.03590 \AA$.

For the numerical simulation a $\mathrm{Si}$ sample based on a simple Si model due to Wooten et al. with 4096 atoms in the unit cell has been used. ${ }^{14}$ The artificial lattice parameter used in the simulation is $a_{1}=45.2402 \AA$. The mass density is $2.323 \mathrm{~g} / \mathrm{cm}^{3}$. The sampling in the real space has been performed on the two-dimensional arrays with dimensions of $1024 \times 1024$ and $2048 \times 2048$. Following Ref. 12 these grid parameters give the adequate sampling for our calculations with real space sampling resolution in the worst case equal to $\Delta x \approx 0.11 \AA /$ pixel, the worst resolution in reciprocal space is $\Delta q \approx 0.01 \AA^{-1}$, and the angular resolution is equal to $\Delta \alpha=\lambda \Delta q \approx 0.2 \mathrm{mrad}^{12}$ The accelerating voltage $V$ is $300 \mathrm{kV}$.

In order to simulate the wave function at the exit face of the specimen we have used the multislice method, ${ }^{12,15,16}$ 

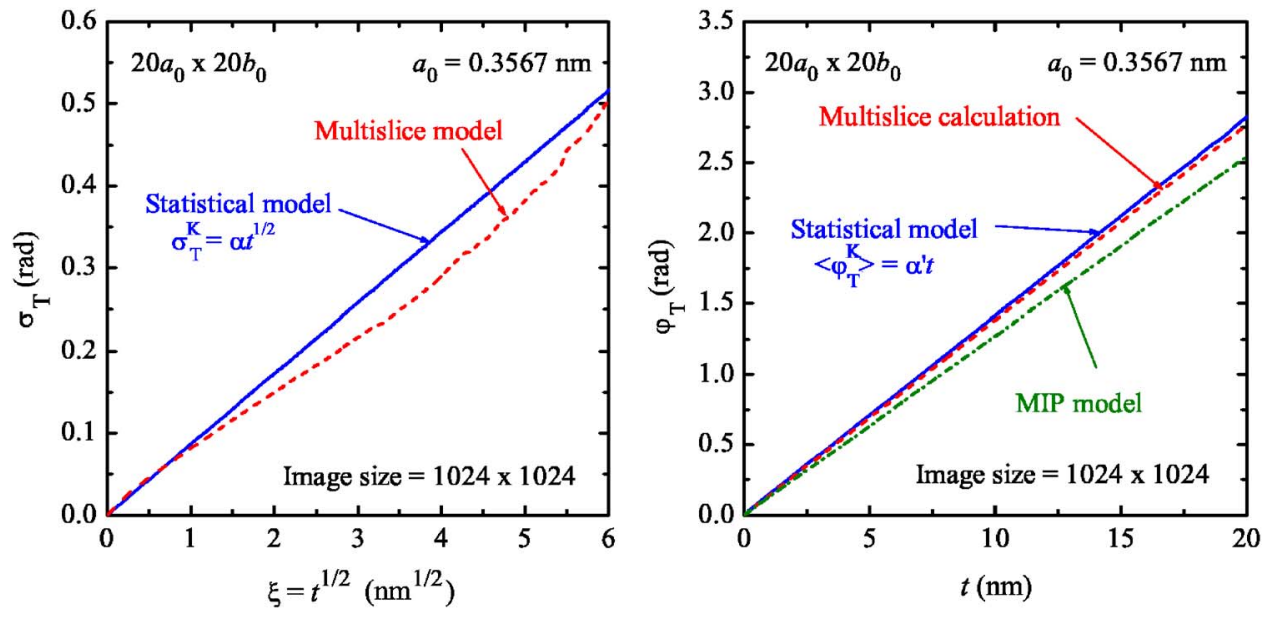

FIG. 2. (Color online) Standard deviation of the exit wave phase and mean phase shift as a function of amorphous carbon sample thickness. which is simpler applicable to amorphous structures as compared with the Bloch wave method, especially for the large artificial unit cells used in the calculation. The plan view transmission electron microscopy setup has been used to simulate the real experiment.

Figure 1 shows the standard deviation of the phase of the exit wave function as a function of the square root of the amorphous Si-sample thickness and the mean value of the exit wave phase as a function of thickness. The numerical simulations have been done with two samples with $2 a_{1} \times 2 a_{1}$ (dotted line) and $4 a_{1} \times 4 a_{1}$ transversal sizes (dashed line). Whereas the analytical form of $\sigma_{T}$ is proportional to $t^{1 / 2}$, the thickness dependence of the standard deviation of the exit wave phase obtained from simulations slightly deviates from this simple law. One can see that the statistical model based on the exit wave phase standard deviation developed in this letter predicts the correct thickness of an amorphous Si sample in the vicinity of $t=25 \mathrm{~nm}$ with an error of $20 \%-25 \%$. The main reason for this is that the developed model does not take into account the quantum mechanical effects in the imaging electron propagation through the specimen. However, these effects can be significant only in the thickness determination model based on the phase standard deviation. The right side of Fig. 1 shows a very small discrepancy in the mean value of the exit wave phase between the numerical simulation and theoretical prediction. In the right side of Fig. 1 the dashed-dotted lines show the results obtained from the experiment (electron holography) and the Hartree-Fock calculations of the mean inner potential (MIP) of the crystalline $\mathrm{Si}^{17,18}$

In order to test the model developed in this letter on another material we have simulated also an experiment with amorphous carbon. The simulated amorphous carbon sample has been obtained from a diamond crystal sample by randomization. Each carbon atom in diamond is surrounded by four nearest neighbors. The lattice parameter used in the simulation is $a_{0}=3.5670 \AA$. The cell volume is $45.385 \times 10^{-24} \mathrm{~cm}^{3}$. The mass density is $3.5155 \mathrm{~g} / \mathrm{cm}^{3}$. The result of the simulation is shown in Fig. 2 (dashed line). In this figure the results of the theoretical model are presented as well (solid line): $\varphi_{T}=0.1385 t$. The result of theoretical model for the mean exit phase shift almost overlaps with that of the multislice simulation. Since the mean inner Coulomb potential for amorphous carbon is available in the literature, ${ }^{19}$ we can compare the predictions of our theory with that based on the carbon mean inner potential. Using the fitting formula given in Eq. (5) of Ref. 19, we obtain the mean inner potential for the amorphous carbon $\langle V\rangle=19.04 \mathrm{eV}$. The result is presented in the right-hand panel of Fig. 2 as a dashed-dotted line.

In conclusion, in this work we have demonstrated a practical method for the determination of the thickness of amorphous samples using statistical data of the phase of the exit wave function.

One of the authors (M.D.C) was grateful to the Fund for Scientific Research-Flanders.

${ }^{1}$ A. Rosenauer, D. Gerthsen, D. Van Dyck, M. Arzberger, G. Böhm, and G. Abstreiter, Ultramicroscopy 51, 11 (2001).

${ }^{2}$ C. Lu, M. J. Lightner, and C. A. Gogal, J. Vac. Sci. Technol. 14, 103 (1977).

${ }^{3}$ J. J. Harris, B. A. Joyce, and P. J. Dobson, Surf. Sci. 103, L90 (1981).

${ }^{4}$ J. Zemek, Acta Phys. Slov. 50, 577 (2000).

${ }^{5}$ S. Terada, H. Murakami, and K. Nishihagi, Semiconductor Europa 2001, Munich, 23 April (unpublished).

${ }^{6}$ C. Wyon, D. Delille, J. P. Gonchond, F. Heider, L. Kwakman, S. Marthon, I. Mazor, A. Michallet, D. Muyard, L. Perino-Gallice, J. C. Royer, and A. Tokar, Thin Solid Films 450, 84 (2004).

${ }^{7}$ C. Liu, J. Erdmann, and A. Macrander, Thin Solid Films 41, 355 (1999).

${ }^{8}$ M. D. Bentzon and P. S. Nielsen, Diamond Relat. Mater. 2, 893 (1993).

${ }^{9}$ G. Stenberg and M. Boman, Diamond Relat. Mater. 5, 1444 (1996).

${ }^{10}$ I. Pozsgai, Ultramicroscopy 107, 191 (2007).

${ }^{11} \mathrm{H}$. Lichte, Handbook of Microscopy, Methods (1997), Vol. 1, pp. 515-536.

${ }^{12}$ E. J. Kirkland, Advanced Computing in Electron Microscopy (Plenum, New York, 1998), Vol. ם, p.

${ }^{13}$ M. Abramowitz and I. A. Stegun, Handbook of Mathematical Functions, Applied Mathematics Series, Vol. - (Dover, New York, 1964), p.

${ }^{14}$ F. Wooten, K. Winer, and D. Weaire, Phys. Rev. Lett. 54, 1392 (1985).

${ }^{15}$ Z. L. Wang, Elastic and Inelastic Scattering in Electron Diffraction and Imaging (Plenum, New York, 1995), Vol. $\mathbf{\square}$, p. $\mathbf{\square}$

${ }^{16}$ D. Van Dyck, J. Microsc. 132, 31 (1983).

${ }^{17}$ M. Schowalter, D. Lamoen, A. Rosenauer, P. Kruse, and D. Gerthsen, Appl. Phys. Lett. 85, 4938 (2004).

${ }^{18}$ P. Kruse, M. Schowalter, D. Lamoen, A. Rosenauer, and D. Gerthsen, Ultramicroscopy 106, 105 (2006).

${ }^{19}$ M. Schowalter, J. T. Titantah, D. Lamoen, and P. Kruse, Appl. Phys. Lett. 86, 112102 (2005). 